MATEC Web of Conferences 22,02008 (2015)

DOI: $10.1051 /$ matecconf/ 20152202008

(C) Owned by the authors, published by EDP Sciences, 2015

\title{
Electromagnetic Modeling of a Linear Synchronous Machine by Lumped Approach
}

\author{
Yang Cui \& Wei Xie \\ College of Logistics Engineering, Shanghai Maritime University, Shanghai, China
}

\begin{abstract}
This paper presents a quantitative analysis method for electromagnetic device called lumped approach. This approach is based on combination of magnetic circuits and finite element method, which provides a good compromise between time calculation and accuracy. The authors propose an electromagnetic model for a linear synchronous machine through this lumped approach. In this research, the linear machine is separated into many small parts. Then, compute all the magnetic reluctances of them with analytical method and acquire the reluctance network. After that the authors couple the electrical part and magnetic part through electromagnetic coupling method. At last, the authors simulate the electromagnetic model of the linear machine and compare the result with that of finite element method.
\end{abstract}

Keywords: electromagnetic device; lumped approach; linear synchronous machine; electromagnetic coupling

\section{INTRODUCTION}

Although the finite element method (FEM) is very accurate, it is not appropriate to all industrial obligations. In a design phase, it is necessary to take the quickness criteria into account. However, an intensive exploration of the design space by the use of optimization methods can lead to prohibitive execution time.

In addition, analytical methods based on the explicit formulation of values characterizing the system have difficulty to consider the local phenomenon and use simplifying assumptions such as the hypothesis of the first harmonic or the hypothesis of linear materials.

The lumped approach (which is semi-analytical or semi-numerical) provides a good compromise between time calculation and accuracy based on magnetic field analysis, and avoids many coarse assumptions and empirical formulas. Therefore, the calculation accuracy and reliability are higher than those of analytical methods which include many coarse assumptions and empirical formulas. And this approach also consumes much less calculation time than FEM. Moreover, it can be well compatible with the design of electromechanical actuators [1] [2].

The lumped approach is based on combination of magnetic circuits and FEM (finite element method). When the model is built with flux-tube method, in this method, the segment in the relatively flux density is even considered to be an element, and the magnetic permeance of each element can be estimated with analytical method. Then, the magnetic permeance network can be set up by connecting each of nodes of permeance according to the analogical method as electrical circuit, and the parameters and performance can be obtained. After that, the magnetic circuit is drawn and we couple it with electrical circuit by a structure like a gyrator, which is called electromag- netic coupling. Finally, the electromagnetic model is established [3] [4] [5].

In this paper, we introduce electromagnetic coupling and how to estimate the permeance with analytical method in the beginning. The main work is to propose an electromagnetic model for a linear synchronous machine by lumped approach. The linear machine is separated into many small parts and we can compute the magnetic reluctance of them with analytical method. Then couple the electrical part and the magnetic part by electromagnetic coupling method to obtain the circuit of the machine. At last, we simulate the electromagnetic model of the linear machine and compare the result with that of finite element method.

\section{ELECTROMAGNETIC COUPLING METHOD}

We use electromagnetic coupling method when build models for electromagnetic device by lumped approach, which can couples the electrical circuit and the magnetic circuit. We use this method to respectively consider electrical circuit and magnetic circuit, so that it is convenient to study and calculate the parameters independently [6].

The electromagnetic coupling is shown as follows:

$$
\begin{aligned}
& \Phi=N I \\
& E=-N \frac{\mathrm{d} \varphi}{\mathrm{d} t}
\end{aligned}
$$

Use (1) and (2) to couple the electrical circuit and the magnetic circuit. Those two formulas are the theoretical condition of electromagnetic coupling when we use lumped approach to build an electromagnetic model. The structure of electromagnetic coupling is like a gyrator. It is shown as follows in Figure 1. 


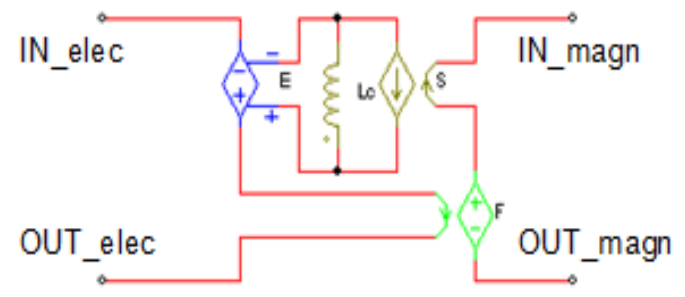

Figure 1. Structure of electromagnetic coupling.

We can see from Figure 1: In the left side of the figure is the electrical part and in the right side is the magnetic part. These two parts are connected by a structure which consists of three dependent sources and an inductor.

The parameters of the dependent sources in this circuit are shown as follows in Table 1.

Table 1. Dependent source of Electromagnetic coupling.

\begin{tabular}{|c|c|c|c|}
\hline Symbol & Component & Category & Gain \\
\hline E & & VCVS & -1 \\
\hline $\mathrm{S}$ & & $\mathrm{CCCS}$ & 1 \\
\hline $\mathrm{F}$ & & CCVS & $N$ \\
\hline
\end{tabular}

There is flux $\varphi$ in the path of magnetic circuit in the right side of Figure 1. Since "S" is a current-controlled current source, and the gain is 1 , it is also the flux $\varphi$ in the path of middle circuit.

$$
V_{\mathrm{L}}=L \frac{\mathrm{d} i_{\mathrm{L}}}{\mathrm{d} t}
$$

In this Formula (3), $V_{\mathrm{L}}$ represents the voltage of inductor " $L$ ". $i_{\mathrm{L}}$ represents the current in this circuit. The value of $i_{\mathrm{L}}$ is equal to $\varphi$ because it is the flux $\varphi$ in the path of middle circuit. So we can use $\varphi$ instead of $i_{\mathrm{L}}$ in (3) and find Formula (4) which is shown as follows:

$V_{\mathrm{L}}=L \frac{\mathrm{d} \varphi}{\mathrm{d} t}$

"E" is a voltage-controlled voltage source and the gain is equal to -1 . Then by linking (4) with (2), we can find that the value of inductor $L$ is equal to $N$.

According to (1), the gain of " $F$ " which is a current-controlled voltage source is equal to $N$. So the magnetomotive force $F$ in the magnetic part in the right side of Figure 1 is equal to NI. $I$ is the current in the left circuit [7].

\section{ESTIMATION OF THE PERMEANCE WITH ANALYTICAL METHOD}

In our research, we have to compute the value of reluctance or permeance in the process of building the electromagnetic model to get the reluctance network of the linear synchronous machine. The machine is separated into many small parts and we can compute the magnetic reluctance of them. This calculation method is based on the geometric of the electromagnetic device. To estimate the reluctance or permeance of a flux tube, we have to calculate with analytical method. It is easy to implement.

We deduce the Formula (5) for calculating reluctances and permeances by this general formula of calculating reluctance:

$R_{\mathrm{m}}=\int_{0}^{L} \frac{\mathrm{d} x}{\mu(x) \cdot S(x)}$

In the Formula (5), $\mu$ means the permeability; L means the integral path; $\mathrm{S}$ means the section.

This formula can be used to analyze the reluctance of all kinds of flux tube's shapes.

Then use this formula to deduce two kinds of flux tubes in our research:

Quarter cylinder is shown in Figure 2.

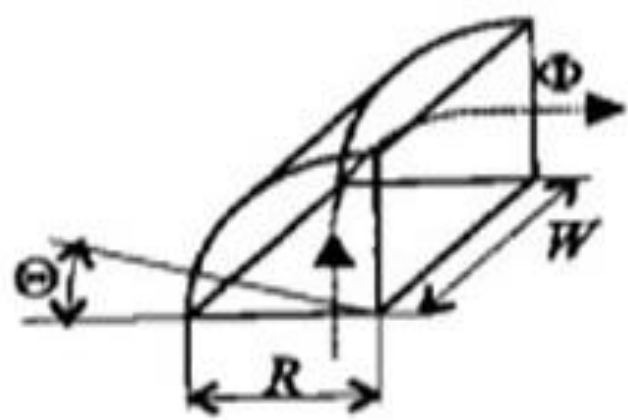

Figure 2. Quarter cylinder.

The reluctance is shown as follows:

$R_{\mathrm{mqc}}=\frac{1}{\mu} \int_{0}^{\pi / 2} \frac{(R / 2) \mathrm{d} \theta}{R \cdot W}=\frac{\pi / 4}{\mu \cdot W}$

The permeance is shown as follows:

$\Lambda_{\mathrm{qc}}=\frac{1}{R_{\mathrm{mqc}}}=\frac{4 \mu W}{\pi}$

In (6) and (7), $R$ means the radius; $W$ means the width.

The rectangular prism is shown in Figure 3. 


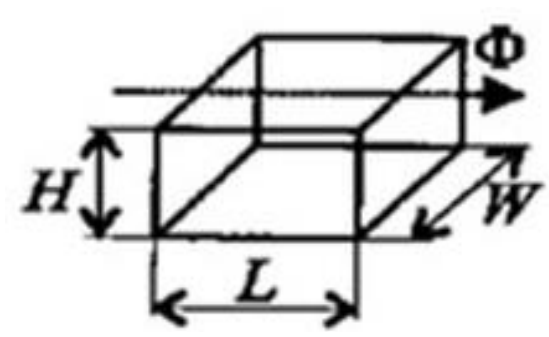

Figure 3. Rectangular prism.

The reluctance is shown as follows:

$R_{\mathrm{mrq}}=\int_{0}^{L} \frac{\mathrm{d} x}{\mu \cdot H \cdot W}=\frac{L}{\mu \cdot W \cdot H}$

The permeance is shown as follows:

$$
\Lambda_{\mathrm{rq}}=\frac{1}{R_{\mathrm{mrq}}}=\frac{\mu W H}{L}
$$

In (8) and (9), $L$ means the length, $W$ means the width and $H$ means the height.

We use these formulas to estimate the reluctance or permeance of the flux tube in the electromagnetic devices. Then, we can obtain the reluctance network and analyze the electromagnetic device. Therefore, we can analyze the rotor and the stator of the linear machine by this method [8].

\section{MODLE OF LINEAR SYNCHRONOUS MA- CHINE}

\subsection{Compute the parameters of rotor and stator}

We set up a model of the linear synchronous machine by lumped approach. The geometry of the machine is shown in Figure 4.
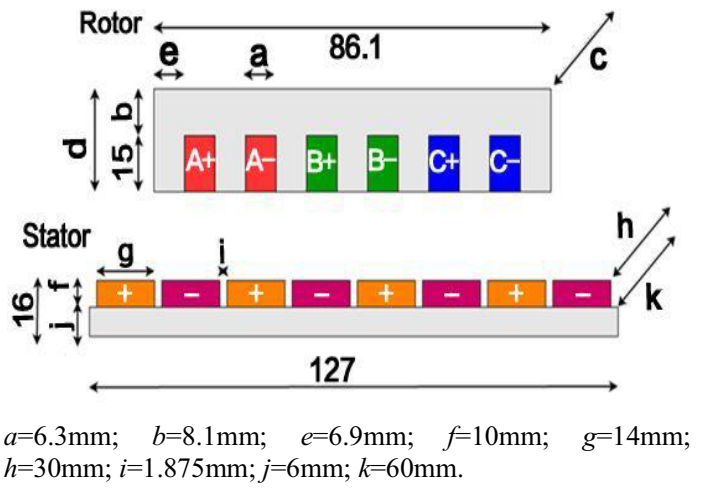

Figure 4. Geometry of the linear synchronous machine. (Unit: $\mathrm{mm})$

Divide the rotor and stator into many small parts and it is convenient to calculate the reluctance of rotor and stator.

The method to divide the rotor and stator is shown in Figure 5.

Rotor:
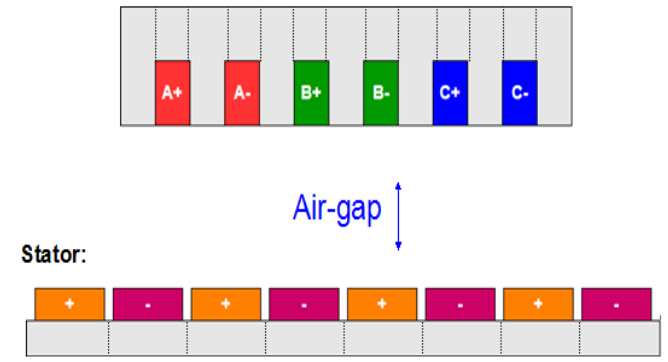

Figure 5. Divide the linear machine.

Each part has its own equivalent magnetic reluctance. Connect all the reluctance and we can obtain the reluctance network of the rotor and the stator, which is respectively shown in Figure 6.

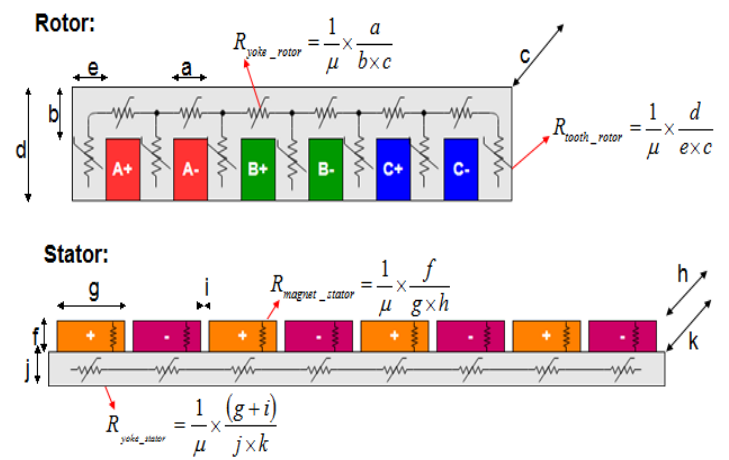

Figure 6. Calculate the reluctances.

Calculate all the reluctance based on (10), which is deduced from (8) as follows:

$$
R=\frac{1}{\mu} \times \frac{L}{S}
$$

In this formula, $\mu$ means the permeability; $S$ means the section; $L$ means the integral path.

We can obtain $(11)(12)(13)(14)$ from (10) shown as follows:

$$
\begin{aligned}
& R_{\text {yoke_rotor }}=\frac{1}{\mu} \times \frac{\mathrm{a}}{\mathrm{b} \times \mathrm{c}} \\
& R_{\text {tooth_rotor }}=\frac{1}{\mu} \times \frac{\mathrm{d}}{\mathrm{e} \times \mathrm{c}} \\
& R_{\text {magnet_stator }}=\frac{1}{\mu} \times \frac{\mathrm{f}}{\mathrm{g} \times \mathrm{h}} \\
& R_{\text {yoke_stator }}=\frac{1}{\mu} \times \frac{\mathrm{g}+\mathrm{i}}{\mathrm{j} \times \mathrm{k}}
\end{aligned}
$$


After calculating all the reluctance in rotor and stator, we can obtain the circuit of the linear machine which is shown in Figure 7. The circuit is composed of the electrical part, the rotor part and the stator part.

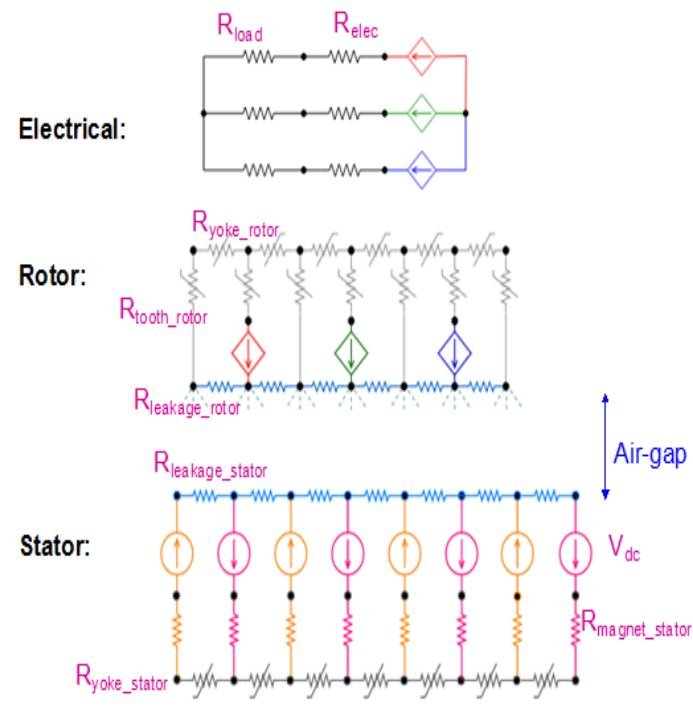

Figure 7. Circuit of the linear machine.

In the circuit, the parameters are shown in Table 2.

Table 2. Parameters of linear machine

\begin{tabular}{|l|l|l|}
\hline Symbols & Explanation & Value \\
\hline$H_{\mathrm{c}}$ & Magnetic field intensity & $800000 \mathrm{~A} / \mathrm{m}$ \\
\hline$B_{\mathrm{r}}$ & Magnetic induction intensity & $1.1 \mathrm{~T}$ \\
\hline$R_{\text {elec }}$ & Electrical resistance & $1.5 \mathrm{Ohm}$ \\
\hline$R_{\text {load }}$ & Load Resistance & $10^{9} \mathrm{Ohm}$ \\
\hline$R_{\text {leakage_rotor }}$ & Leakage resistance of rotor & $10^{9} \mathrm{H}^{-1}$ \\
\hline$R_{\text {leakage_stator }}$ & Leakage resistance of stator & $10^{9} \mathrm{H}^{-1}$ \\
\hline$N s$ & Number of turns & 210 \\
\hline
\end{tabular}

According to the formula as follows:

$F=H_{\mathrm{c}} \times L$

In the formula (15): $F$ is the magnetic motive force; $H_{\mathrm{c}}$ is the magnetic field intensity; $L$ is the length of the device. Therefore, we can obtain $F_{\mathrm{dc}}$, which is the magnetomotive force of the stator tooth:

$F_{\mathrm{dc}}=H_{c} \times \mathrm{f}=800000 \times 0.01=8000 \mathrm{~A}$

We use electromagnetic coupling method to connect the electrical part and rotor part as shown in Figure 7 (dependent source). It can help us respectively analyze the electrical circuit and the magnetic circuit
[9].

\subsection{Compute the resistors in the air-gap}

In the air gap, we need to connect each rotor tooth to each stator tooth. Thus, each rotor point has eight reluctances. For example:

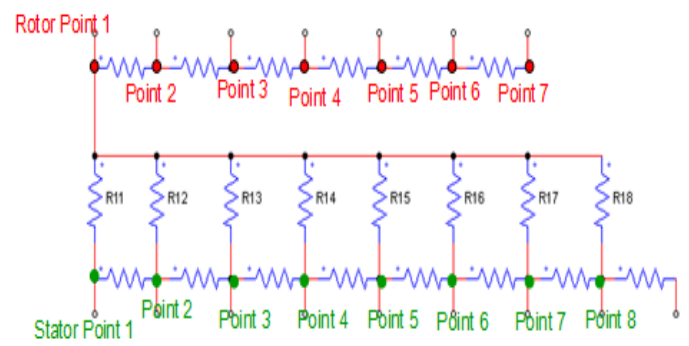

Figure 7. Reluctances on Rotor Point 1

Figure 7 shows the reluctances of Rotor Point 1. We name the reluctances of air gap according to this principle: If the reluctance connects Rotor Point 1 and Stator Point 1, then the reluctance is called 'R11'. Similarly, if the reluctance connects Rotor Point 1 and Stator Point 2, then the reluctance is called 'R12'and so on. So we can obtain the circuit of the air gap as follows:

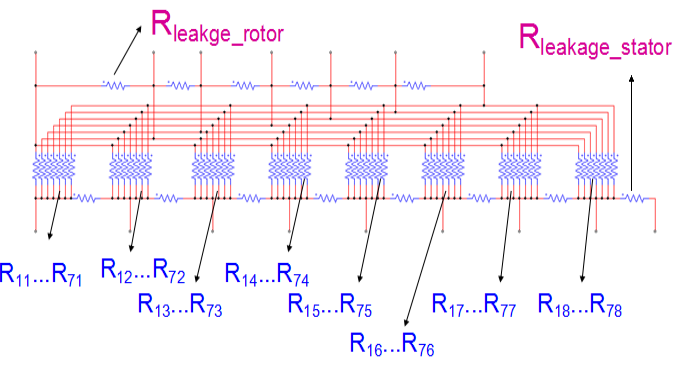

Figure 8. Reluctances in the air gap.

We draw this circuit and name all the reluctances. It is to be presented in the bell-shaped function (16) as follows:

$R_{\mathrm{g}}=\left[\frac{\alpha}{1+\left|\frac{x-\gamma}{\beta}\right|^{2 \delta}}\right]^{-1}$

In this function, $R_{\mathrm{g}}$ is the values of reluctances in the air gap, and $\alpha, \beta, \delta$ are given by other researches that are "Studies of the evolution of air-gap permeance". $X$ is the relative position of the rotor and the stator [10].

In Figure 9: $T_{\mathrm{r}}$ represents the distance between two neighboring teeth of rotor, which is equal to $13.2 \mathrm{~mm}$; $T_{\mathrm{s}}$ represents the distance between two neighboring 
teeth of stator, which is equal to $15.875 \mathrm{~mm}$.

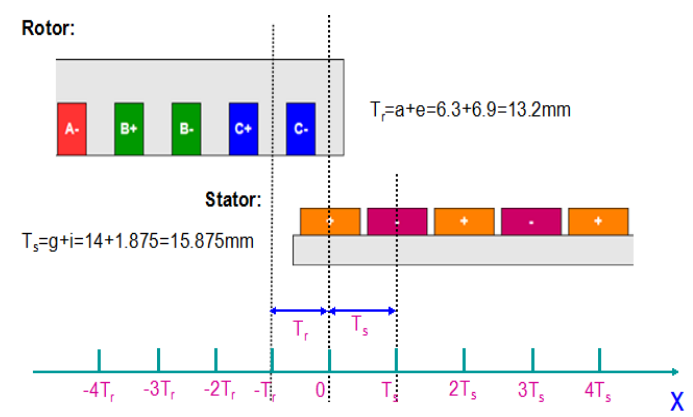

Figure 9. Position of the machine.

The rotor moves to the right, and the speed is $2 \mathrm{~mm}$ per second.

The $\gamma_{11}$ between rotor 1 and stator 1 is 0

The $\gamma_{12}$ between rotor 1 and stator 2 is $T_{\mathrm{s}}$.

The $\gamma_{21}$ between rotor 2 and stator 1 is $T_{\mathrm{r}}$.

The $\gamma_{22}$ between rotor 2 and stator 2 is $T_{\mathrm{r}}+T_{\mathrm{s}}$.

Calculate each $\gamma$ of reluctance. After that we obtain this Table 3 about each $\gamma$.

Table 3. Value of $\gamma$ in the air gap

\begin{tabular}{|l|l|l|l|l|}
\hline $\boldsymbol{\gamma}$ & Stator1 & Stator2 & $\ldots$ & Stator8 \\
\hline Rotor1 & 0 & $1 T \mathrm{~s}$ & $\ldots$ & $7 T \mathrm{~s}$ \\
\hline Rotor2 & $1 T \mathrm{r}$ & $T \mathrm{~s}+T \mathrm{r}$ & $\ldots$ & $7 T \mathrm{~s}+T \mathrm{r}$ \\
\hline Rotor3 & $2 T \mathrm{r}$ & $T \mathrm{~s}+2 T \mathrm{r}$ & $\ldots$ & $7 T \mathrm{~s}+2 T \mathrm{r}$ \\
\hline Rotor4 & $3 T \mathrm{r}$ & $T \mathrm{~s}+3 T \mathrm{r}$ & $\ldots$ & $7 T \mathrm{~s}+3 T \mathrm{r}$ \\
\hline Rotor5 & $4 T \mathrm{r}$ & $T \mathrm{~s}+4 T \mathrm{r}$ & $\ldots$ & $7 T \mathrm{~s}+4 T \mathrm{r}$ \\
\hline Rotor6 & $5 T \mathrm{r}$ & $T \mathrm{~s}+5 T \mathrm{r}$ & $\ldots$ & $7 T \mathrm{~s}+5 T \mathrm{r}$ \\
\hline Rotor7 & $6 T \mathrm{r}$ & $T \mathrm{~s}+6 T \mathrm{r}$ & $\ldots$ & $7 T \mathrm{~s}+6 T \mathrm{r}$ \\
\hline
\end{tabular}

The parameters $\alpha, \beta$ and $\delta$ are obtained from other researches which are "Studies of the evolution of air-gap permeance". This topic is studied by my classmate Fan $\mathrm{Hu}$. The topic is to implement finite-element method and analytical method for obtaining the evolution of the air-gap permeance, and it is linked with my topic on the linear machine. Thus the method used in the topic is FEM and my method is a lumped approach. After calculating permeance points during the research of the topic, the researcher of this topic finds out a bell-shaped curve which is closest to all permeance points as the permeance curve (the evolution of permeance), which can get $\alpha, \beta$ and $\delta$ :

$$
\alpha=2.4379 * 10^{-7} ; \beta=0.00641 ; \delta=1.476
$$

Therefore, we can calculate all the reluctances in the air gap from Formula (12). Build the whole electromagnetic model of the linear synchronous machine which consists of the electrical part, the rotor part and the stator part. In this model: The electrical part and the rotor part are connected by electromagnetic coupling; the rotor part and the stator part are connected through an air-gap reluctance network.

\subsection{Simulation of linear machine}

Simulate this electromagnetic model of the linear synchronous machine, which is set up by lumped approach and compare the result with that of finite element method. Compute the electromotive force of $E_{b}$ in the electrical circuit in Figure 10. Ignore hysteresis loss and eddy current loss.

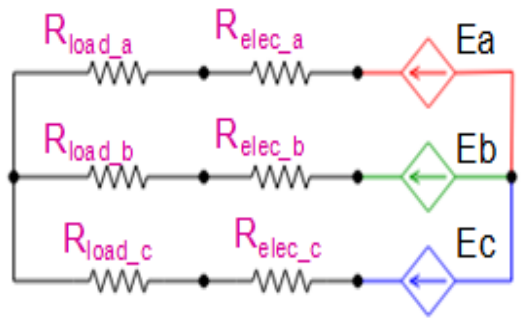

Figure 10. Electrical part of linear machine.

The result of simulation is shown in Figure 11:
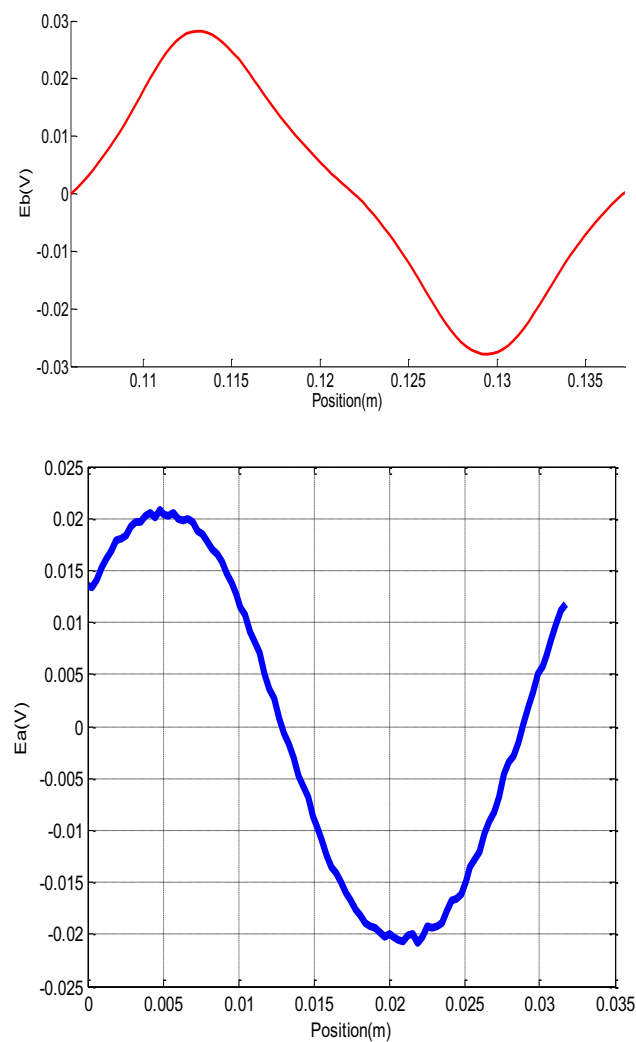

Figure 11. Electromotive force of Eb. 
The first figure of Figure 11 shows the result by lumped approach; the second figure shows that by FEM. We can see that in the first figure the amplitude of the electromotive force of $\mathrm{E}_{\mathrm{b}}$ is about $0.028 \mathrm{~V}$; the cycle is $0.03 \mathrm{~m}$. While in the second figure, the amplitude of the electromotive force of $E_{b}$ is about $0.021 \mathrm{~V}$; the cycle is also $0.03 \mathrm{~m}$. The results are similar.

Then compute the magnetic flux through $E_{b}$ in the magnetic circuit of rotor in Figure 12.

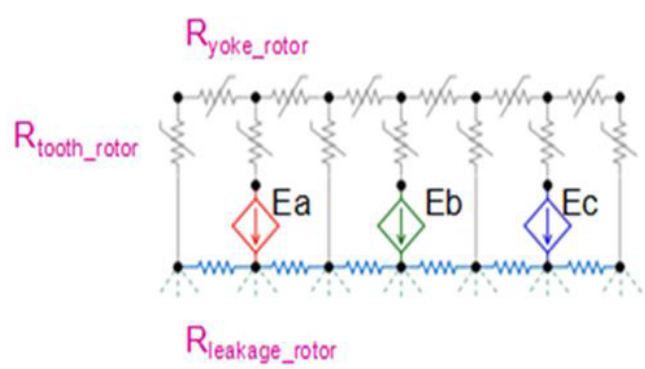

Figure 12. Magnetic circuit of Rotor.
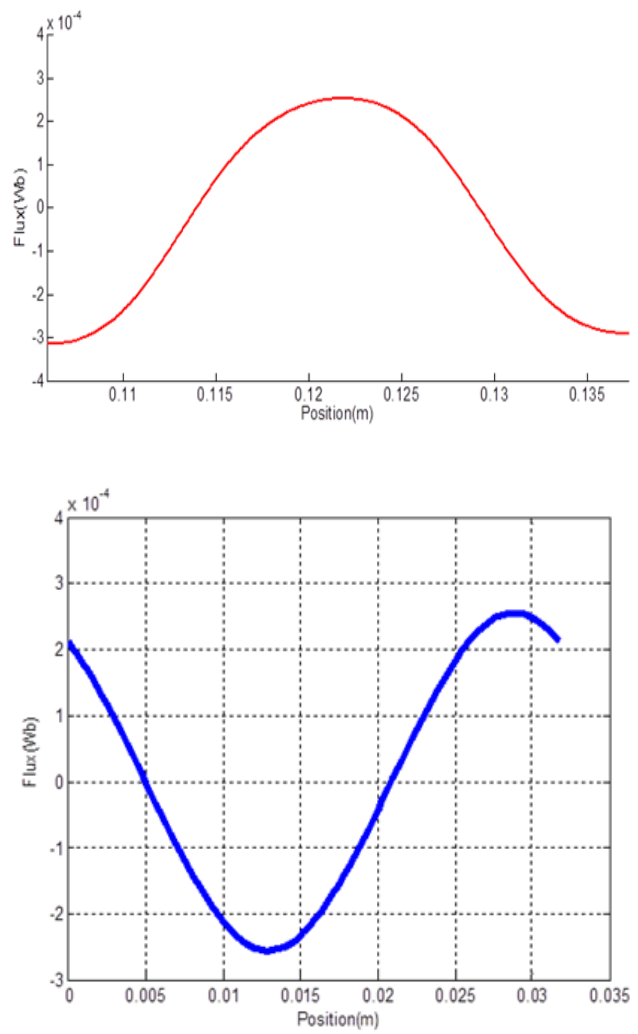

Figure 13. Flux through Eb.

The first figure of Figure 13 shows the result by lumped approach; the second figure shows that by FEM. We can see that in the first figure, the amplitude of the magnetic flux through $\mathrm{E}_{\mathrm{b}}$ is about $0.00025 \mathrm{~Wb}$; the cycle is about $0.03 \mathrm{~m}$. While in the second figure, the amplitude of the magnetic flux through $\mathrm{E}_{\mathrm{b}}$ is also about $0.00025 \mathrm{~Wb}$; the cycle is about $0.03 \mathrm{~m}$ similarly. The results are almost the same.

\section{CONCLUSION}

We present a method of quantitative analysis for electromagnetic device, which is called lumped approach. We set up an electromagnetic model for a linear synchronous machine by this approach. The linear machine is separated into many small parts. Then compute all the magnetic reluctances of them with analytical method and obtain the reluctance network. After that, we combine the magnetic circuit with electrical one by using electromagnetic coupling method and analyze the air-gap reluctance network. At last, we simulate the electromagnetic model of the linear machine and compare the result with that of finite-element method.

We can find that the result of lumped approach is very close to that of FEM. It is convenient to compute parameters and set up a model by this lumped approach. Using this method, we can quickly build a model of electromagnetic device even if the structure and condition are more complex.

\section{ACKNOWLEDGMENT}

The authors would like to express their gratitude to all those who helped them during the writing of this paper.

The authors are very grateful to their classmate Fan $\mathrm{Hu}$. He gave them the parameters in the Bell-Shaped Function during their research, which helps them obtain result successfully.

The authors are very grateful to their teacher Nicolas Bracikovski because he gave them constant encouragement and guidance.

\section{References}

[1] Kenji Nakamura, Osamu Ichinokura, Mitsuru Maeda, Shigeaki Akatsuka, Kazuo Takasugi, Hiromichi Sato. 2000. Analysis of orthogonal-core type linear variable inductor and application to VAR compensator. IEEE Transactions on Magnetics, 36(5): 3565-3567.

[2] Kenji Nakamura, Kenichi Saito, and Osamu Ichinokura. 2003. Dynamic analysis of interior permanent magnet motor based on a magnetic circuit model. IEEE Transactions on Magnetics, 39(5): 3250-3252.

[3] Kenji Nakamura, Shinya Fujio, Osamu Ichinokura. 2006 A method for calculating iron loss of an SR motor based on reluctance network analysis and comparison of symmetric and asymmetric excitation. IEEE Transactions on Magnetics, 42(10): 3440-3442. 
[4] Gilles Guedia Guemo, Patrice Chantrenne, Julien Jac. 2013. Application of classic and T lumped parameter thermal models for permanent magnet synchronous machines. IEEE Transactions on Magnetics, pp: 809-815.

[5] Gilles Guedia Guemo, Patrice Chantrenne, Julien Jac. 2013. Parameter identification of a lumped parameter thermal model for a permanent magnet synchronous machine. IEEE Transactions on Magnetics, $\mathrm{pp}$ : 1316-1320.

[6] Kenji Nakamura, Shuichi Hayakawa, Shigeaki Akatsuka, Takashi Ohinata, Kazuo Minazawa, Osamu Ichinokura. 2005. Three-dimensional reluctance network analysis considering an iron loss characteristic for an EIE-Core Variable Inductor. IEEE Transactions on Magnetics, 41(10): 4033-4035.

[7] O. Ichinokura, S. Suyama, T. Watanabe, H. J. Guo. 2001. A new calculation model of switched reluctance motor for use on spice. IEEE Transactions on Magnetics, 37(4): 2834-2836.

[8] Kenji Nakamura, Shinya Fujio, Osamu Ichinokura. 2006. A Method for Calculating Iron loss of a Switched Reluctance Motor Based on Reluctance Network Analysis, Graduate School of Engineering, Sendai; Tohoku University, pp: 1021-1026.

[9] A. A. Aboulnaga, A. Emadi. 2004. Simplified Simulation and Modeling Technique for Integrated Magnetic Components in Power Electronic Converters, Grainger Power Electronics and Motor Drives Laboratory, Electrical Power and Power Electronics Center, Illinois Institute of Technology Chicago, pp: 725-730.

[10]Nicolas Bracikowski, Michel Hecquet, Pascal Brochet, Sergey V. Shirinskii. 2012. Multi-physics modeling of a permanent magnet synchronous machine by using lumped models. IEEE Transactions on Industrial Electronics, 59(6): 2426-2437. 